\title{
Fosfowash: Early proof of concept study investigating intravesical fosfomycin for recurrent urinary tract infections
}

\author{
Blayne Welk, MD, MSc'; Christopher Munday, PhD2; Jeremy Burton, MSc, PhD'; Richard J. Baverstock, MD \\ 'Department of Surgery and Epidemiology and Biostatistics, Western University, London, ON, Canada; ${ }^{2}$ Lawson Research Institute, London, ON, Canada; ${ }^{3}$ Departments of Surgery and Microbiology \& \\ Immunology, Western University, London, ON, Canada; ${ }^{4}$ Department of Surgery, Cumming School of Medicine, University of Calgary, AN, Canada
}

Cite as: Welk B, Munday C, Burton J, et al. Fosfowash: Early proof of concept study investigating intravesical fosfomycin for recurrent urinary tract infections. Can Urol Assoc J 2021;15(12):423-4. http://dx.doi.org/10.5489/cuaj.7365

Published online June 22, 2021

\section{Introduction}

Patients with neurogenic lower urinary tract dysfunction (NLUTD) rate urinary tract infections (UTIs) as one of the most bothersome problems. ${ }^{1}$ This is a frustrating, multifactorial problem that often arises due to the use of catheters, abnormal bladder compliance, coexisting neurogenic bowel dysfunction, multidrug-resistant organisms, and specific deficiencies in host defense mechanisms. ${ }^{2}$ The management of NLUTD patients who are plagued by an abnormally high frequency or severity of UTIs and are using clean intermittent catheters $(\mathrm{CIC})$ may require tertiary treatment options to try and prevent UTIs. Clinicians often struggle with the potential harms of continuous oral antibiotic prophylaxis (such as antibiotic resistance) and this approach is often considered a last resort. ${ }^{3,4}$

Intravesical antibiotic bladder instillation is an alternative option that is well-suited to patients already using CICs. Previous studies have generally focused on the use of gentamicin:5-7 however, it an expensive medication, it is cumbersome to obtain for patients, and in recent years, it has been the victim of manufacturing delays and drug shortages. Our objective was to investigate if fosfomycin tromethamine, a readily available oral antibiotic that comes in powder form, may be a suitable agent for intravesical bladder instillation therapy.

\section{Methods}

Fosfomycin tromethamine is a bactericidal antibiotic with a broad range of activity against gram-positive and negative aerobic bacteria (such as Escherichia coli, Enterococcus, Klebsiella, Proteus, Serratia, pseudomonas, and Citrobacter) that are commonly associated with UTIs. It has in vitro anti- microbial activity against extended-spectrum beta-lactamase (ESBL)-producing bacteria and can penetrate biofilms. It inactivates the enolpyruvyl transferase enzyme (which inhibits bacterial cell wall synthesis) and reduces adherence of bacteria to the urothelium. ${ }^{8,9}$

We conducted two in vitro experiments using fosfomycin. We tested three solutions: reconstituted fosfomycin (created by mixing a commercially available package of fosfomycin tromethamine [JAMP Pharma Corporation, QC, Canada] in $125 \mathrm{~mL}$ of distilled water), tobramycin $160 \mathrm{mg}$ in $1 \mathrm{~L}$ normal saline (positive control), and buffered saline (negative control).

The objective of our first experiment was to determine if reconstituted fosfomycin tromethamine was bactericidal in the absence of metabolism by the human gut (where it is normally metabolized to its free acid, fosfomycin). We added 10 $\mu \mathrm{L}$ of the fosfomycin solution to a tryptic soy agar (TSA) plate of $10^{5}$ colony-forming units (CFU) of urinary isolate, E. coli 67, and the plate was incubated at $37^{\circ} \mathrm{C}$ aerobically overnight.

The objective of our second experiment was to determine the minimum inhibitory concentration of the fosfomycin and tobramycin solutions; $1 \mathrm{~mL}$ of each of the solutions was added to $1.5 \mathrm{~mL}$ Eppendorf tubes in triplicate using the following dilutions based on the original solutions $(100 \%$, $50 \%, 25 \%$, and $12.5 \%$ ). Then $1 \times 10^{5}$ CFU of E. coli 67 was added to each tube. Tubes were incubated over night at $37^{\circ} \mathrm{C}$ and agitated at $170 \mathrm{rpm}$. Bacterial survival was assessed the next day by dilution series and drop-plating on TSA plates.

\section{Results}

The fosfomycin solution had a similar $\mathrm{pH}(5.5)$ to the tobramycin solution (6.5). In the first experiment, fosfomycin and tobramycin were effective at inhibiting the growth of $E$. coli 67 , with a zone of inhibition of $31 \mathrm{~mm}$ and $20 \mathrm{~mm}$, respectively. This result was replicated after one week of storage at $4^{\circ} \mathrm{C}$, showing that there is no loss of efficacy over this time period (data not shown). In our second experiment, all four dilutions of both the tobramycin and the fosfomycin inhibited all bacterial growth of E. coli 67; the negative control series had high growth of bacteria (Fig. 1). 


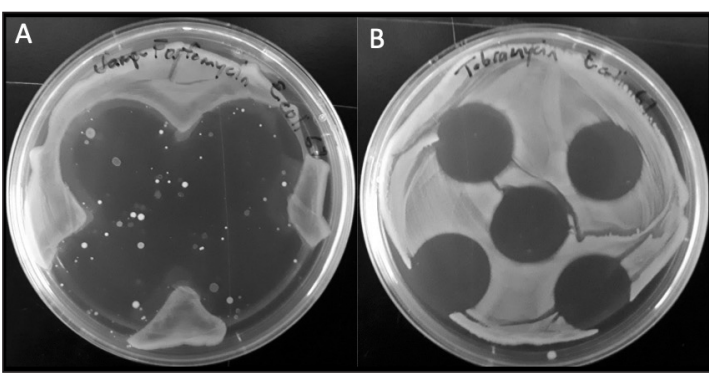

Fig. 1. Fosfomycin at the concentration tested was effective at inhibiting the growth of E. coli 67 with a zone of inhibition that was similar to that of tobramycin. Results of fosfomycin are shown in panel $A$, and tobramycin in panel $B$.

\section{Discussion}

A recent systematic review identified 11 previous studies of intravesical antibiotic therapy and found that this approach was generally efficacious at both the treatment and prevention of UTIs, with minimal side effects. ${ }^{10}$ This conclusion supports the need to develop further feasible intravesical options. To our knowledge, this is the first investigation into the use of fosfomycin as an intravesical antibiotic. We have shown promising, preliminary data that support the further investigation of fosfomycin as an intravesical antibiotic agent for patients with NLUTD and frequent UTIs. As both the fosfomycin and tobramycin achieved complete bacterial kill in all the tested dilations, we know the minimal inhibitory concentration for E. coli 67 is less than a $12.5 \%$ dilution (equivalent to one satchel of commercial fosfomycin in $1 \mathrm{~L}$ of water).

Ideally, intravesical antibiotics are instilled before bedtime, at the time of the last catheter of the day and allowed to dwell overnight. Potential advantages of fosfomycin as a bladder instillation include the ability of patients to obtain it from any outpatient pharmacy, the ease of storing a reconstituted satchel in the fridge for use over the course of a week (for example, reconstituting in a $500 \mathrm{~mL}$ or $1000 \mathrm{~mL}$ of commercial bottled water), and the wide antimicrobial spectrum of fosfomycin. Fosfomycin has often been used as a once-per-week oral therapy for recurrent UTIs, however, using it as an intravesical therapy may have benefits of avoiding systemic side effects, reducing bacterial resistance in the gut flora, and improved patient acceptance compared to continuous oral use. Three patients with NLUTD who were unable to access intravesical aminoglycosides due to the current pandemic switched to using fosfomycin instillations due to lack of alternatives. Anecdotally, they found it to be tolerable and to have a similar efficacy to their previous tobramycin instillations.

Limitations of this work include the use of a single UTIrelevant bacterial strain, no direct assessment of potential cytotoxic effects (although it is typically well-tolerated orally), and the potential for resistance to develop with longterm use. Further basic science and clinical studies will be necessary to determine clinical efficacy and safety and to test different dilution options and instillations schedules.

Competing interests: The authors do not report any competing personal or financial interests related to this work.

This paper has been peer-reviewed.

\section{References}

1. Pannek J. Prevention of recurrent urinary tract infections in neurourology. Eur Urol Focus 2020;6:817-9. https://doi.org/10.1016/i.euf.2020.01.015

2. Kennelly $M$, Thiruchelvam $N$, Averbeck $M A$, et al. Adult neurogenic lower urinary tract dysfunction and intermittent catheterization in a community setting: Risk factors model for urinary tract infections. Adv Urol 2019;2019:2757862-13. https://doi.org/10.1155/2019/2757862

3. Fisher $\mathrm{H}$, Oluboyede $\mathrm{Y}$, Chadwick T, et al. Continuous low-dose antibiotic prophylaxis for adults with repeated urinary tract infections (AnTIC): A randomized, open-label trial. Lancet Infect Dis 2018;18:95768. https://doi.org/10.1016/S1473-3099(18)30279-2

4. Blaser M. Antibiotic overuse: Stop the killing of beneficial bacteria. Nature 2011;24;476:393-4. https://doi.org/10.1038/476393a

5. Abrams $P$, Hashim H, Tomson C, et al. The use of intravesical gentamicin to treat recurrent urinary tract infections in lower urinary tract dysfunction. Neurourol Urodyn 2017;36:2109-16. https://doi.org/10.1002/nau.23250

6. Cox L, He C, Bevins J, et al. Gentamicin bladder instillations decrease symptomatic urinary tract infections in neurogenic bladder patients on intermittent catheterization. Can Urological Assoc J 2017;11:350-4. https://doi.org/10.5489/cuaj.4434

7. Stalenhoef JE, Nieuwkoop C van, Menken PH, et al. Intravesical gentamicin treatment for recurrent urinary tract infections caused by multidrug resistant bacteria. J Urol 2019;201:549-55. https://doi.org/10.1016/i.juro.2018.10.004

8. Falagas ME, Vouloumanou EK, Samonis G, et al. Fosfomycin. Clin Microbiol Rev 2016;29:321-47. https://doi.org/10.1128/CMR.00068-15

9. Maraki S, Samonis G, Rafailidis PI, et al. Susceptibility of urinary tract bacteria to fosfomycin. Antimicrob Agents Ch 2009;53:4508-10. hitps://doi.org/10.1128/AAC.00721-09

10. Pietropaolo A, Jones $P$, Moors $M$, et al. Use and effectiveness of antimicrobial intravesical treatment for prophylaxis and treatment of recurrent urinary tract infections (UTIS): A systematic review. Curr Urol Rep 2018;19:78. https://doi.org/10.1007/s1 1934-018-0834-8

Correspondence: Dr. Blayne Welk, Department of Surgery and Epidemiology and Biostatistics, Western University, London, 0N, Canada; bkwelk@gmail.com 